\title{
High-Resolution Mid-Infrared Spectral Reconstruction using a Subwavelength Coaxial Aperture Array
}

\author{
Benjamin Craig ${ }^{1}$, Jiajun Meng ${ }^{2}$, Vivek Raj Shrestha ${ }^{1}$ Jasper J. Cadusch ${ }^{2}$, and Kenneth B. Crozier ${ }^{1,2}$ \\ ${ }^{1}$ School of Physics, University of Melbourne, Victoria 3010, Australia \\ ${ }^{2}$ Department of Electrical and Electronic Engineering, University of Melbourne, Victoria 3010, Australia \\ Corresponding Author e-mail address: kenneth.crozier@unimelb.edu.au
}

\begin{abstract}
We demonstrate mid-infrared computational spectroscopy using an array of coaxial aperture filters. We experimentally determine material transmission spectra using an algorithm whose inputs are the transmission spectra and the power transmitted through each filter. OCIS codes: (250.5403) Plasmonics; (300.6340) Spectroscopy, infrared; (080.1753) Computation methods
\end{abstract}

\section{Introduction}

One approach to miniaturize spectrometers involves pairing a filter array with a detector array. It has been shown that the spectrum of light illuminating such a device can be determined by reconstruction algorithms that take as inputs the signals (e.g. photocurrents) measured from the detectors in the array and the transmission spectra of the filters. Devices for the visible and near-infrared spectrum have been demonstrated [1-3]. Recently, proof-of-principle demonstrations have been made in the mid-infrared [4,5], but high-resolution reconstruction has not been shown. In this work, we fabricate a collection of 101 mid-infrared pass-band filters, each comprising an array of subwavelength coaxial apertures in a gold $\mathrm{(Au}$ ) film on a $\mathrm{Si}$ wafer. Each filter has a transmission peak, whose spectral position we tune from $6.2 \mu \mathrm{m}$ to $14.2 \mu \mathrm{m}$ by appropriate choice of geometry. Here, we use these filters to perform a proof-ofprinciple demonstration of reconstructing the spectrum of an infrared light source (silicon carbide globar). We furthermore reconstruct the spectra of the globar after transmission through various polymer materials. This allows determination of the transmission spectra of these materials. We show that the transmission spectra reconstructed in this way are in good agreement with those directly measured by our Fourier transform infrared (FTIR) microscope.

\section{Filter Design and Fabrication}

Each filter is a square array of coaxial apertures (annular vacancies) in an Au film (140 nm thick, with $2 \mathrm{~nm}$ of Cr for adhesion), with an overall extent of $\sim 100 \times 100 \mu \mathrm{m}$. The substrate is a double-side polished undoped Si wafer. The filters are fabricated by e-beam lithography, e-beam evaporation and lift-off. Fig. 1a. illustrates the unit cell schematic. In our collection of filters, the period $P$ varies from $1.7 \mu \mathrm{m}$ to $3.7 \mu \mathrm{m}$. For each filter, the inner $\left(R_{1}\right)$ and the outer radii $\left(R_{2}\right)$ are set to be $0.25 \times P$ and $1.4 \times R_{1}$ respectively. An SEM image of the filter with $P=1.7 \mu m$ is shown as Fig. 1b. An optical microscope image of several filters is shown in Fig. 1c.

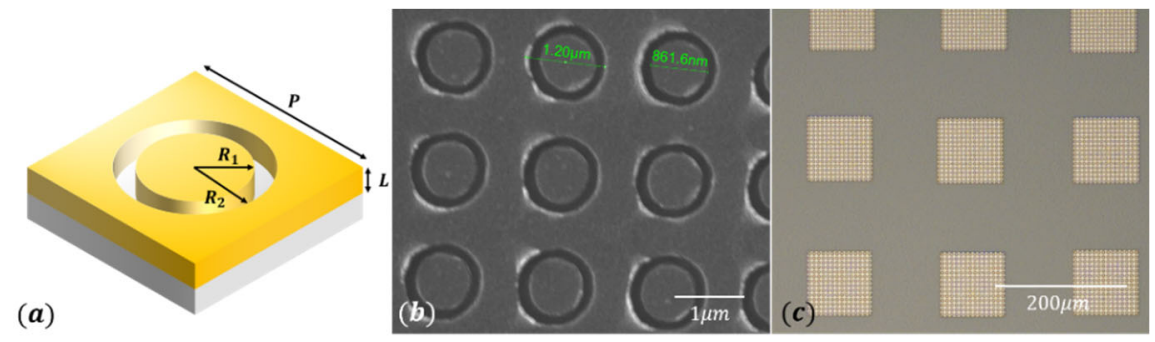

Fig. 1. (a) Schematic of coaxial aperture unit cell. (b) SEM image of $1.7 \mu \mathrm{m}$ period filter. (c) Microscope image of several filters.

\section{Results}

The transmission functions of the filters are measured using an FTIR microscope (PerkinElmer/Spotlight). From Fig. 2a, it is seen that measured and simulated spectra are in good agreement (for filter with $P=1.7 \mu \mathrm{m}$ ), both in resonance position and peak transmission. In our simulations, the filter array is illuminated through the Si substrate, at an angle of $10^{\circ}$ at the Si-air interface. This is done for the following reason. The reflecting objective of the FTIR has a numerical aperture of $N A=0.6$, corresponding to illumination angles up to $\sim 36^{\circ}$. The filters are illuminated through the underside of the Si substrate (n 3.4), meaning that the light transmitted into the Si substrate is incident on the Si-air interface at an angle up to $\sim 10^{\circ}$. The fact that the simulations assume that the light is incident at $\sim 10^{\circ}$ 
while in experiments a distribution of angles is inevitable might account for the discrepancies between simulation and experiments. Fabrication imperfections may also contribute. Fig. 2b. shows measured absolute transmission spectra for all the 101 filters. Fig. 2c. shows a comparison of the resonance position between simulated and measured devices.
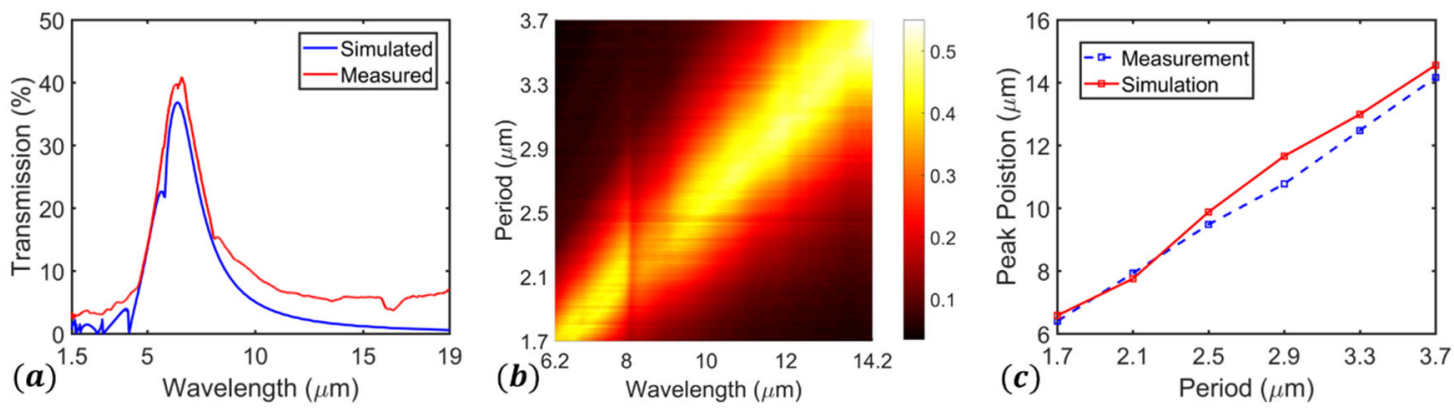

Fig. 2. (a) Comparison between simulated and measured absolute transmission for the $P=1.7 \mu \mathrm{m}$ filter. (b) Measured transmission for all 101 filters. (c) Comparison of simulated and measured peak positions of filters.

Next, we demonstrate spectral reconstruction using the filter array. Ideally, the filter array would be combined with a detector array. At the time of writing, we do not have an appropriate detector array available to us, so we instead measure the light transmitted through each filter with our FTIR microscope, to emulate what would be measured by each detector of the detector array. The spectral reconstruction is performed as follows. The transmission spectrum of each filter $T_{i}(\lambda)$ is measured by the FTIR microscope, representing a column of the filter matrix $\boldsymbol{F}(2801 \times 101)$. Next the total optical signal $S_{i}(\lambda)$ transmitted through each filter is measured. This represents $S_{i}(\lambda)=$ $\int G(\lambda) \times M(\lambda) \times T_{i}(\lambda) \times M C T_{\text {resp }}(\lambda) d \lambda$, where $G(\lambda)$ is the globar (IR light source) power density spectrum, $M(\lambda)$ is the transmission spectrum of the test material (placed between the globar and the filters) and $M C T_{\text {resp }}(\lambda)$ is the responsivity of the mercury cadmium telluride (MCT) detector of our FTIR microscope. The transmitted signals form the $S$-vector $(1 \times 101)$. The filter matrix $F$ and the signal vector $S$ are then input into our implementation of the recursive least squares (RLS) method. This estimates $I^{M}(\lambda)=G(\lambda) \times M(\lambda) \times M C T_{\text {resp }}(\lambda)$. The transmission spectrum $M(\lambda)$ of the material being tested is determined by dividing $I^{M}(\lambda)$ by $I(\lambda)=G(\lambda) \times M C T_{\text {resp }}(\lambda) . I(\lambda)$ is the reconstruction of the globar spectrum without no material between the globar and filters. $M(\lambda)$ reconstructed for several materials can be seen in Fig. 3. The results shown are in good agreement with the spectra measured directly by the FTIR. To quantify this, we calculate the normalised mean absolute error (NMAE). This is the mean of the absolute difference between the spectrum measured by the FTIR and that reconstructed by our system, normalised to the peak signal value. These spectra exhibit finer features than [4,5], thereby expanding the relevance of this approach.
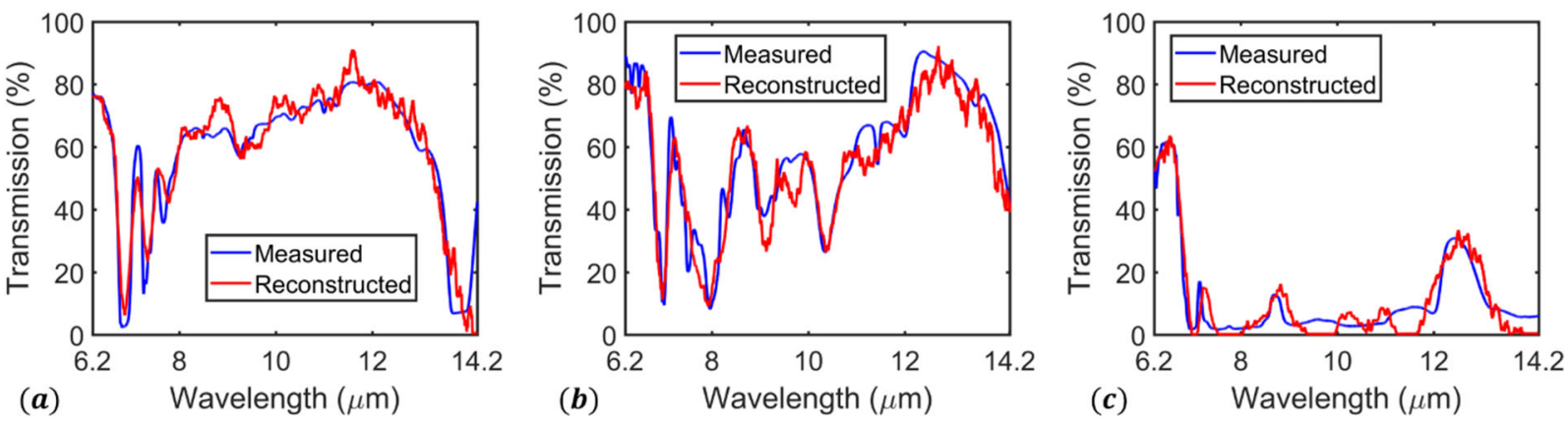

Fig. 3. Reconstruction of the material transmission spectra $M(\lambda)$ for (a) polyethylene (NMAE $=0.0590)$, (b) cellophane $(0.0703)$ and $(\mathbf{c})$ polyvinyl chloride $(\mathrm{NMAE}=0.0605)$.

\section{References}

[1] J. Bao and M. G. Bawendi, Nature 523, 67 (2015)

[2] E. Huang, Q. Ma and Z. Liu, Sci. Rep. 7, 40693 (2017)

[3] S. W. Wang, C. Xia, X. Chen, W. Lu, M. Li, H. Wang, W. Zhang and T. Zheng, Opt. Lett. 32, 632 (2007)

[4] B. Craig, V. R. Shreshtha, J. Meng, J. J. Cadusch, K. B. Crozier, Opt. Lett. 43, 4481 (2018)

[5] A. Wang and Y. Dan, Sci. Rep. 8, 11257 (2018)

Performed in part at Melbourne Centre for Nanofabrication (MCN) in Victorian Node of Australian National Fabrication Facility. Supported in part by DARPA (HR0011-16-1-004), by VESKI, and by the Australian Research Council (DP150103736, DP180104141 and FT140100577). 


\section{University Library}

\section{- M M N E R VA A gateway to Melbourne's research publications}

Minerva Access is the Institutional Repository of The University of Melbourne

Author/s:

Craig, B;Meng, J;Shrestha, VR;Cadusch, JJ;Crozier, KB

Title:

High-resolution mid-infrared spectral reconstruction using a subwavelength coaxial aperture array

Date:

2019-01-01

Citation:

Craig, B., Meng, J., Shrestha, V. R., Cadusch, J. J. \& Crozier, K. B. (2019). High-resolution mid-infrared spectral reconstruction using a subwavelength coaxial aperture array. CLEO: Applications and Technology 2019, Part F127-CLEO_AT 2019, OSA \& IEEE. https:// doi.org/10.1364/CLEO_AT.2019.JTu2A.48.

Persistent Link:

http://hdl.handle.net/11343/294882 